\title{
EDITORIAL
}

\section{UK Resuscitation Council guidelines on emergency treatment of anaphylactic reactions: a primary care perspective}

Anaphylactic emergencies are uncommon in primary care, but the correct immediate response and appropriate followup can save lives. ${ }^{1,2}$ Unfortunately, practitioners can be uncertain about both management and prevention of anaphylactic emergencies. ${ }^{3-6}$ The latest guideline on managing anaphylaxis by the UK Resuscitation Council ${ }^{7}$ carries several important messages for primary care, both in the UK and beyond. Firstly, it aids recognition by making explicit what anaphylaxis is - and isn't - and grounds its emergency management in the ABCDE (Airway, Breathing, Circulation, Disability, Exposure) framew ork that characterises the Resuscitation Council response to medical and traumatic emergencies. Secondly, it tackles confusion about treatment, streamlining recommendations for drug and non-drug management, and making it clear that generalists should not use intravenous adrenaline. Thirdly, it recommends that patients who have an episode of anaphylaxis should be followed up by a specialist in allergy.

Assessment using the ABCDE approach should improve recognition. Sudden onset and rapid progression of lifethreatening symptoms is characteristic of anaphylaxis. Airway signs (difficulty breathing or swallowing, feeling the throat closing, hoarseness and/or stridor) are the first priority for recognition, followed by Breathing (respiratory rate, wheeze) and Circulation (hypotension, shock) signs, resulting in Disability (confusion, agitation, loss of consciousness), with skin and mucosal changes under $\mathrm{E}$ for exposure. The guideline is explicit that "skin changes without lifethreatening airway, breathing or circulation problems do not signify an anaphylactic reaction". For most primary care staff dealing with infrequent allergic emergencies, clarity about what is and isn't anaphylaxis (or, in practice, when intramuscular adrenaline should and should not be used) is critically important. In line with other Resuscitation Council documents this guideline also includes the recurring mantra: "get help early on".

Acting promptly and correctly is also vital, and the guidelines contain an anaphylaxis algorithm to aid practitioners. The changes in treatment are subtle, and are geared towards ease of implementation. The doses of adrenaline now come in just three age bands: under six years - 150 micrograms; six to 12 years (or puberty) - 300 micrograms; and teenagers and adults - 500 micrograms. The preferred route is explicitly stated: intramuscular - i.e. using a blue (23G) needle by default into the anterolateral mid-thigh at 90 degrees. The guideline reminds about using intravenous fluids if access is available, and lying the patient as flat as their breathing permits in order to minimise hypotension and empty ventricle syndrome. Advice about intravenous adrenaline is clear; for anaphylaxis it should only ever be administered, with cardiac monitoring, by specialists in emergency medicine or anaesthesia.

This is a good guideline. The problem is, however, that even good guidelines are in themselves ineffective at changing the way most clinicians work. To make a difference, this guideline needs to be incorporated into practice at several levels. For individual clinicians, effective guidelines must be readily to hand, particularly in emergencies. Primary care staff in their first few years of practice, and those who treat allergic emergencies regularly, should memorise the doses of adrenaline; the rest of us should accept our fallibility and write them in our diary, and emergency drug boxes, as well as pinning the algorithm to the clinic wall. But effective practise also needs rehearsal. Practices and educational organisations should make sure that anaphylaxis training is included in the annual cardiopulmonary resuscitation refresher which all UK primary care staff who give immunisations should undertake. Education which involves multidisciplinary teams enacting an emergency scenario including talking to the imaginary patient and being embarrassed by missing something out in front of colleagues - is more effective than simply reading and discussing the guideline. Again, the use of the ABCDE approach should make this step easier.

Finally, organisations that commission care need to address the issue of specialist input following an episode of anaphylaxis. The guideline recommends referral of patients with anaphylaxis to an allergy clinic for advice on long-term 
management and, crucially, prevention of emergencies. Given the inequitable distribution of specialist allergy clinics in the UK, this is not always an option, and while more fully accredited specialists in allergy are needed, it is not appropriate for patients and organisations to wait for them to appear. The guideline does not specify who or what the specialist should be, and commissioning organisations should consider managed clinical networks or using intermediate grades with appropriate training - such as GPs with special interests and clinical nurse specialists in allergy - to deliver long-term follow-up and support.

\section{References}

1. Panesar SS, Walker S, Sheikh A. Primary care management of anaphylaxis. Prim Care Resp J 2003;12:124-6.

2. Simons FER. Anaphylaxis, killer allergy: long-term management in the community. J Allergy Clin Immunol 2006;117:367-77.

3. Hayman GR, Bansal JA, Bansal AS. Knowledge about using auto-injectable adrenaline: review of patients' case notes and interviews with general practitioners. BMJ 2003;327:1328.

4. Walker S, Sheikh A. Managing anaphylaxis: effective emergency and long-term care are necessary. Clin Exp Allergy 2003;33:1015-18.

5. Akeson N, Worth A, Sheikh A. The psychosocial impact of anaphylaxis on young people and their parents. Clin Exp Allergy 2007;37:1213-20.

6. M cLean-Tooke A, Bethune C, Fay A, Spickett G. Adrenaline in the treatment of anaphylaxis: what is the evidence? BMJ 2003;327:1332-5.

7. Resuscitation Council (UK). Emergency treatment of anaphylactic reactions. Guidelines for healthcare providers. Resuscitation Council (UK). London, 2008. http://www.resus.org.uk/pages/reaction.pdf

\section{Christopher Burton \\ Senior Research Fellow}

\section{*Allison Worth}

Research Fellow

Allergy and Respiratory Research Group

Division of Community Health Sciences: General Practice Section University of Edinburgh,

20 West Richmond Street,

Edinburgh, EH8 9DX

* Correspondence:

Tel: +44 (0)131 6509463

Fax: +44 (0)131 6509119

E-mail: Allison Worth@ed.ac.uk

13th M arch 2008

\section{Available online at http://www.thepcrj.org}

